\title{
QCD fits \& Factorisation tests in diffraction at HERA
}

A next-to-leading-order QCD analysis of diffractive processes measured by the ZEUS experiment

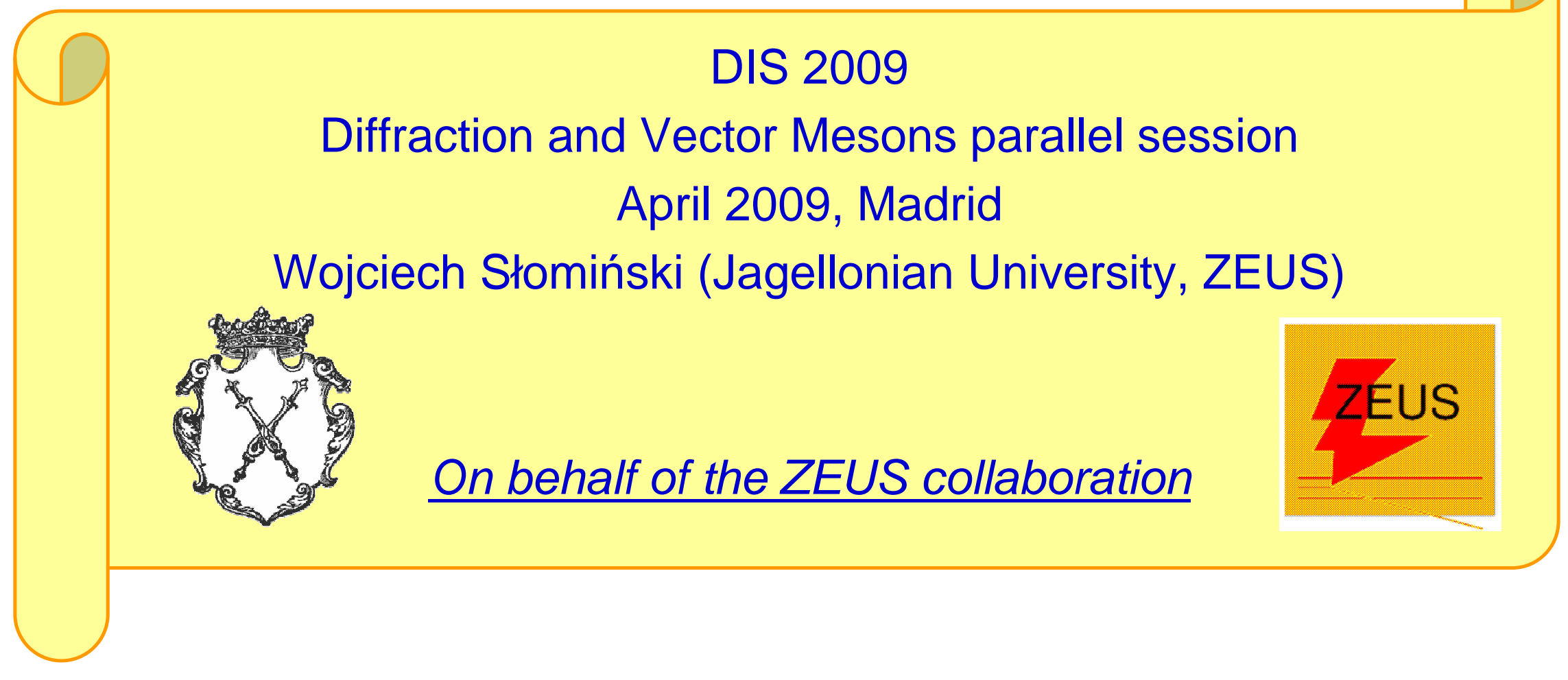




\section{Fitting \& testing procedure}

- A systematic investigation

- NLO QCD fit to the inclusive DIS data

- comparison and fit to dijets in DIS

- comparison to dijets in PHP

- Massive quarks treatment

- VFNS vs. FFNS

- arguments for GM-VFNS

- Fixing gluons 


\section{Inclusive diffractive DIS}

Kinematics of diffractive deep inelastic scattering

$\beta=\frac{Q^{2}}{2\left(p-p^{\prime}\right) q} \approx$ parton in IP fract. momentum

$t$-integrated cross section

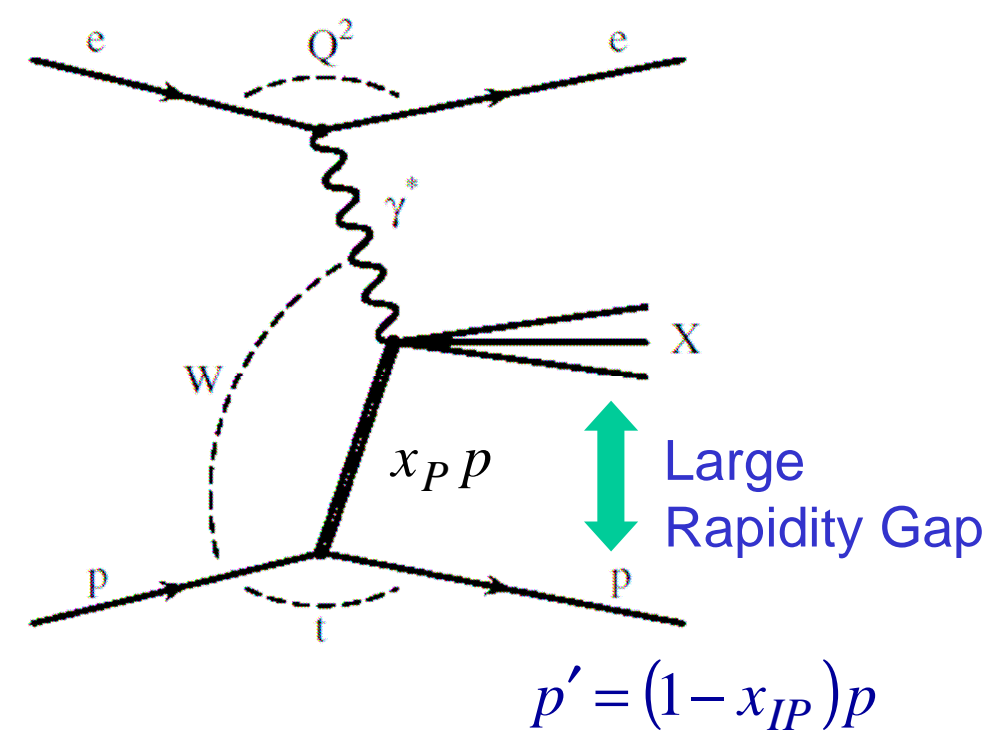

$$
\frac{d \sigma}{d \beta d Q^{2} d x_{\mathbb{P}}}=\frac{2 \pi \alpha^{2}}{\beta Q^{4}}\left(1+(1-y)^{2}\right) \sigma_{\mathrm{r}}^{D(3)}\left(\beta, Q^{2}, x_{\mathbb{P}}\right)
$$

expressed in terms of diffractive structure functions

$$
\sigma_{\mathrm{r}}^{D(3)}\left(\beta, Q^{2}, x_{\mathbb{P}}\right)=\underline{F_{2}^{D(3)}\left(\beta, Q^{2}, x_{\mathbb{P}}\right)}-\frac{y^{2}}{1+(1-y)^{2}} F_{\mathrm{L}}^{D(3)}\left(\beta, Q^{2}, x_{\mathbb{P}}\right)
$$




\section{A model for diffractive $F_{2 / L}$}

\section{Regge factorisation assumption}

$$
F_{2 / \mathrm{L}}^{D(4)}\left(\beta, Q^{2}, x_{\mathbb{P}}, t\right)=f_{\mathbb{P}}\left(x_{\mathbb{P}}, t\right) F_{2 / \mathrm{L}}^{\mathbb{P}}\left(\beta, Q^{2}\right)+f_{\mathbb{R}}\left(x_{\mathbb{P}}, t\right) F_{2 / \mathrm{L}}^{\mathbb{R}}\left(\beta, Q^{2}\right)
$$

This assumption works for the inclusive DIS with

- Regge-type flux $f\left(x_{\mathbb{P}}, t\right)=\frac{A e^{B t}}{x_{\mathbb{P}}^{2 \alpha(t)-1}}$ with $\alpha(t)=\alpha(0)+\alpha^{\prime} t$

- $\quad$ ree $F_{2 / \mathrm{L}}^{\mathbb{P}}\left(\beta, Q^{2}\right)$

- $F_{2 / \mathrm{L}}^{\mathbb{R}}\left(\beta, Q^{2}\right) \propto F_{2 / \mathrm{L}}^{\pi}\left(\beta, Q^{2}\right)$ (GRV) see Marta Ruspa talk

\section{Can QCD + DGLAP describe $F_{2 / L}^{\mathbb{P}}\left(\beta, Q^{2}\right)$ ?}




\section{Factorisation \& heavy quarks}

- $F_{2 / L}$ or any other cross section $\sigma$ for $N$ massless flavours

- collinear divergencies caused by massless quarks factorised and absorbed into non-perturbative PDFs

$$
\sigma\left(Q^{2}, \ldots\right)=\sum_{k} f_{k}^{(N)}\left(Q^{2}\right) \otimes \sigma_{k}(\ldots)
$$

- nb. in diffraction

$$
f_{k}^{(N)}\left(Q^{2}\right) \rightarrow f_{k}^{D(N)}\left(Q^{2}, x_{I P}, t\right)
$$

- + 1 heavy flavour (massive quark) in FFNS

$$
\sigma\left(Q^{2}, \ldots\right)=\sum_{k} f_{k}^{(N)}\left(Q^{2}\right) \otimes \sigma_{k}^{\mathrm{FF}}\left(m^{2} / Q^{2}, \ldots\right)
$$

- still $N$ partons, heavy flavour in final state only

- no extra collinear divergencies

- new types of terms

- $m^{2} / Q^{2}$ - important at low $Q^{2}$

- $\log \left(m^{2} / Q^{2}\right)$ - large at high $Q^{2}$ 


\section{Heavy quarks treatment in VFNS}

- $m^{2} / Q^{2} \rightarrow 0$ massless or infinite $Q^{2}$ limit

- large logs must be resummed

$\rightarrow \mathrm{N}+1$ massless flavours

$$
\sigma\left(Q^{2}, \ldots\right)=\sum_{k} f_{k}^{(N+1)}\left(Q^{2}\right) \otimes \sigma_{k}(\ldots)
$$

- ZM(zero mass)-VFNS

- use $(\mathrm{N}+1)$ massless formula at $\mathrm{Q}^{2}>\mathrm{m}^{2}$

- $\mathrm{GM}($ general mass)-VFNS

- $\log \left(m^{2} / Q^{2}\right)$ resummed $\rightarrow$ heavy quark PDF

- proper behaviour at $Q^{2} \sim m^{2}$

$$
\sigma\left(Q^{2}, \ldots\right)=\sum_{k} f_{k}^{(N+1)}\left(Q^{2}\right) \otimes \sigma_{k}^{\mathrm{VF}}\left(m^{2} / Q^{2}, \ldots\right)
$$

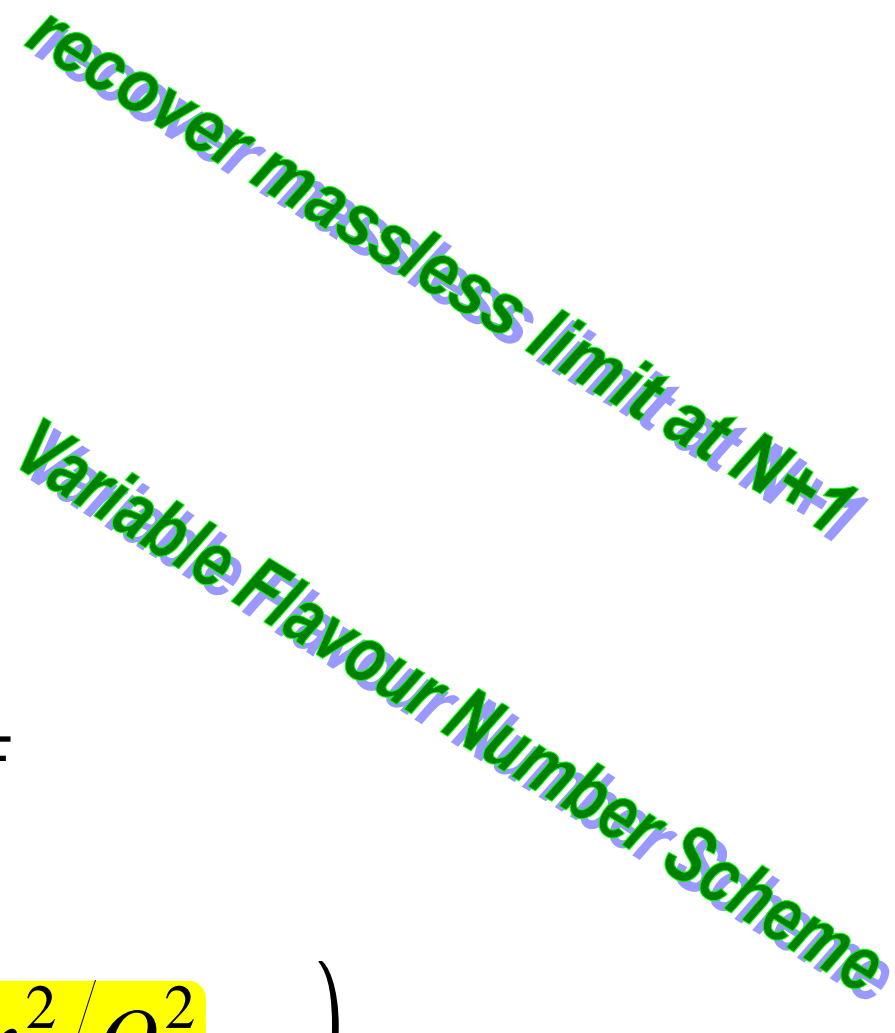

- non-unique - Thorne-Roberts scheme used (as in ZEUS QCD fits) 


\section{FN scheme choice}

- GM-VFNS is most general $\rightarrow$ best choice

- Inclusive DIS

- FFNS and VFNS formulae available

- both schemes give good description of the data

- Dijets production

- available formulae (computer codes) use massless quarks

- "closer" to VFNS than FFNS

- VFNS provides heavy quark PDFs

- OK at high $\mu$

- still threshold effects missing at $\mu$ close to $m_{h}$ 


\section{Diffractive PDFs parametrization}

\section{Regge factorisation assumption}

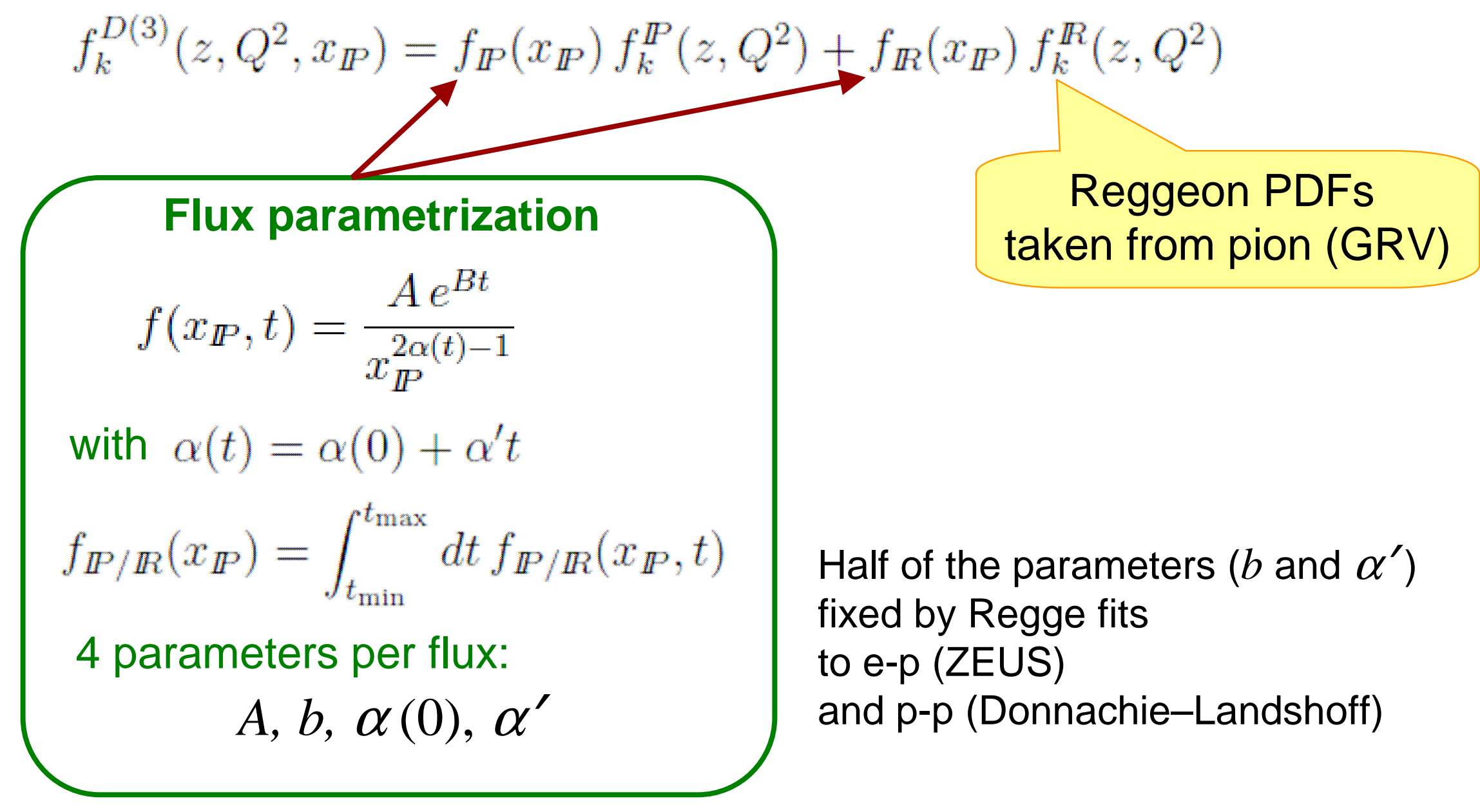




\section{Pomeron PDFs parametrization}

Pomeron PDFs parametrized at some initial $\mathrm{Q}_{0}{ }^{2}$

for all flavours $q=q$

$\Rightarrow$ quark singlet (total sea) $f_{S}^{I P}=\sum_{q} f_{q^{+}}^{I P}=2 \sum_{q} f_{q}^{I P}$

symmetric light quarks assumed: $d=u=s$

$z f_{k}^{I P}\left(z, Q_{0}^{2}\right)=A_{k} z^{B_{k}}(1-z)^{C_{k}} \quad k=\mathrm{g}, \mathrm{S} \quad 6$ parameters

× regularizing factor $\exp \left(-\frac{0.001}{1-z}\right)$ to allow for any $C_{k}$

Free flux parameters: $\alpha_{P}(0), \alpha_{R}(0), A_{R}$

3 parameters

\section{9 parameters in total}




\section{Models for gluons}

Gluons expected to be poorly constrained by the inclusive data.

Consider two cases of the gluon parametrization

$$
z f_{g}^{I P}\left(z, Q_{0}^{2}\right)=A_{g} z^{B_{g}}(1-z)^{C_{g}}
$$

"Standard": Fit $\mathrm{S}$ with $B_{g}, C_{g}$ fitted

"Constant": Fit $\mathrm{C}$ with $B_{g}=C_{g}=0$ (as in H1-2006B)

Both models provide equally good data description

but

very different gluons 


\section{LRG data well described - low $Q^{2}$}

\section{ZEUS}

- ZEUS LRG 99-00 — ZEUS (prel.) DPDF $S$ incl - - - ZEUS (prel.) DPDF $S$ incl (extrapolated)

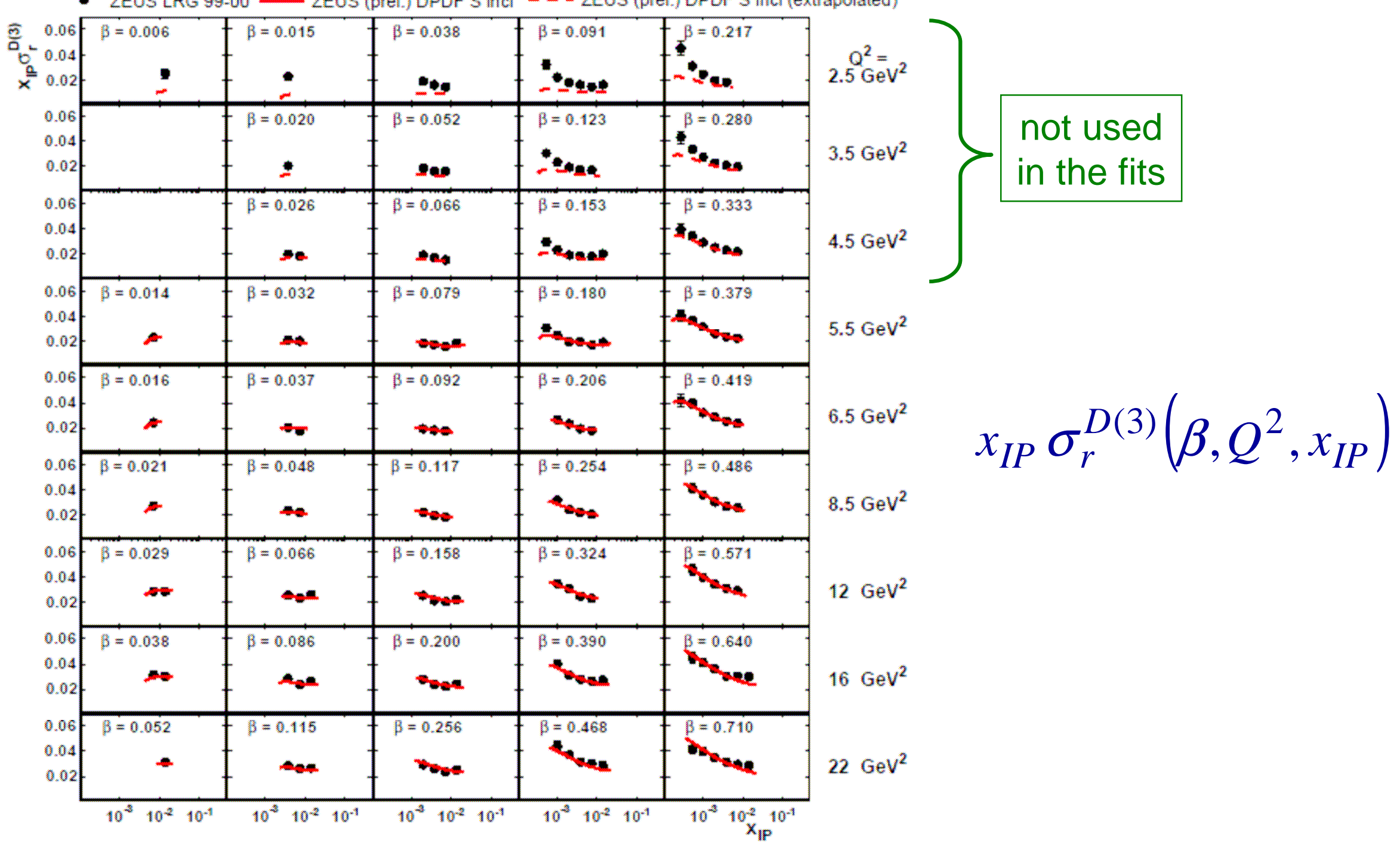




\section{LRG data well described - high $Q^{2}$}

\section{ZEUS}

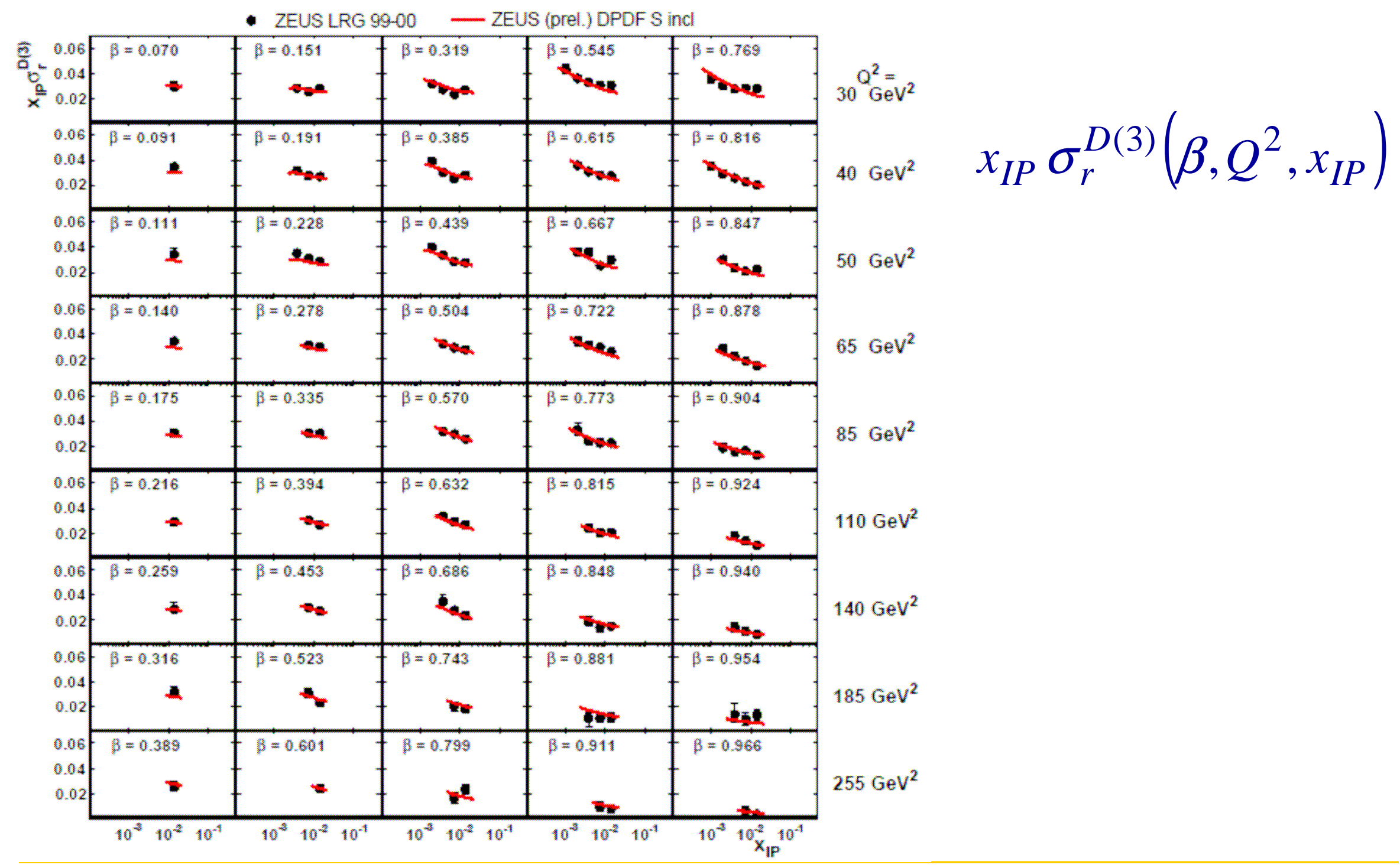




\section{LPS data well described}

$$
x_{I P} \sigma_{r}^{D(3)}\left(\beta, Q^{2}, x_{I P}\right)
$$

\section{ZEUS}

- ZEUS LPS 99-00 - ZEUS (prel.) DPDF S incl - - - ZEUS (prel.) DPDF S incl (extrapolated)

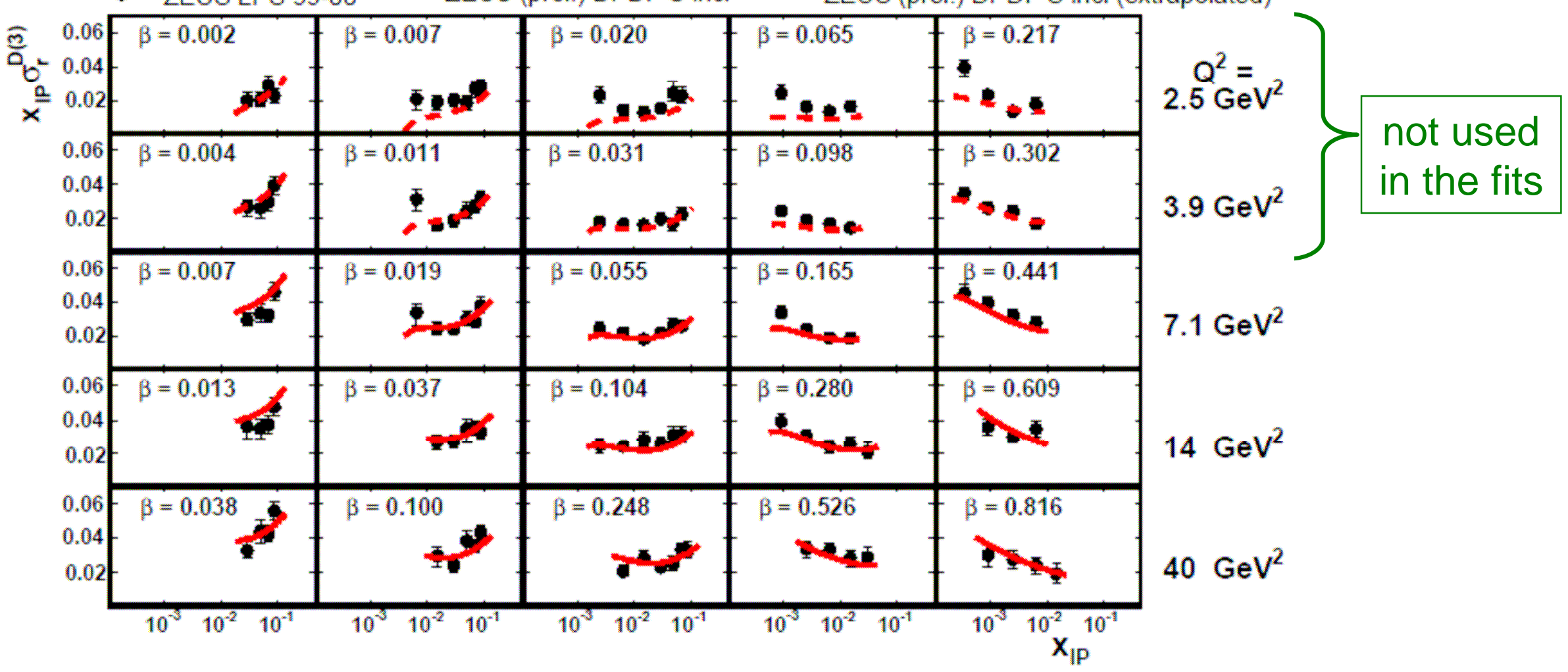




\section{DPDFs from the inclusive fits}

\section{ZEUS}
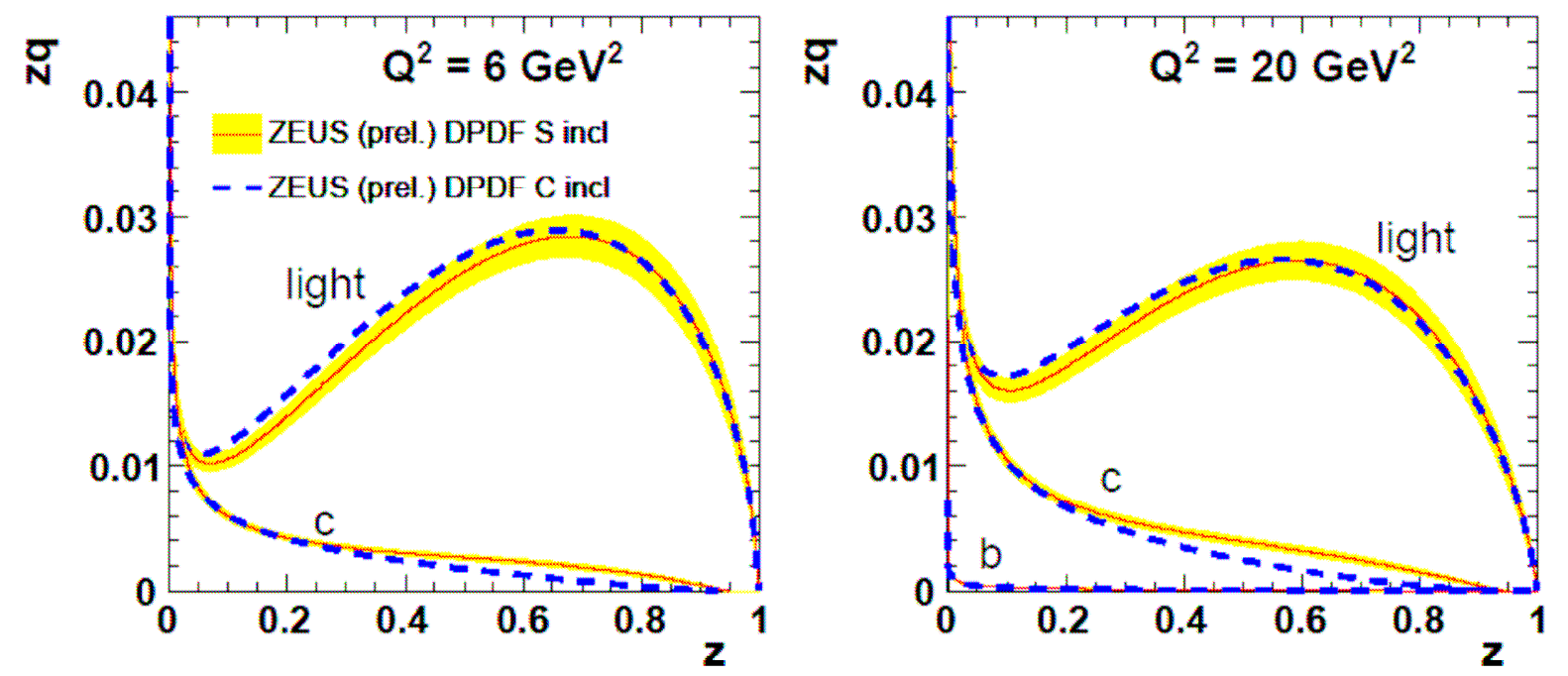

yellow band shows fit experimental uncertainty

N
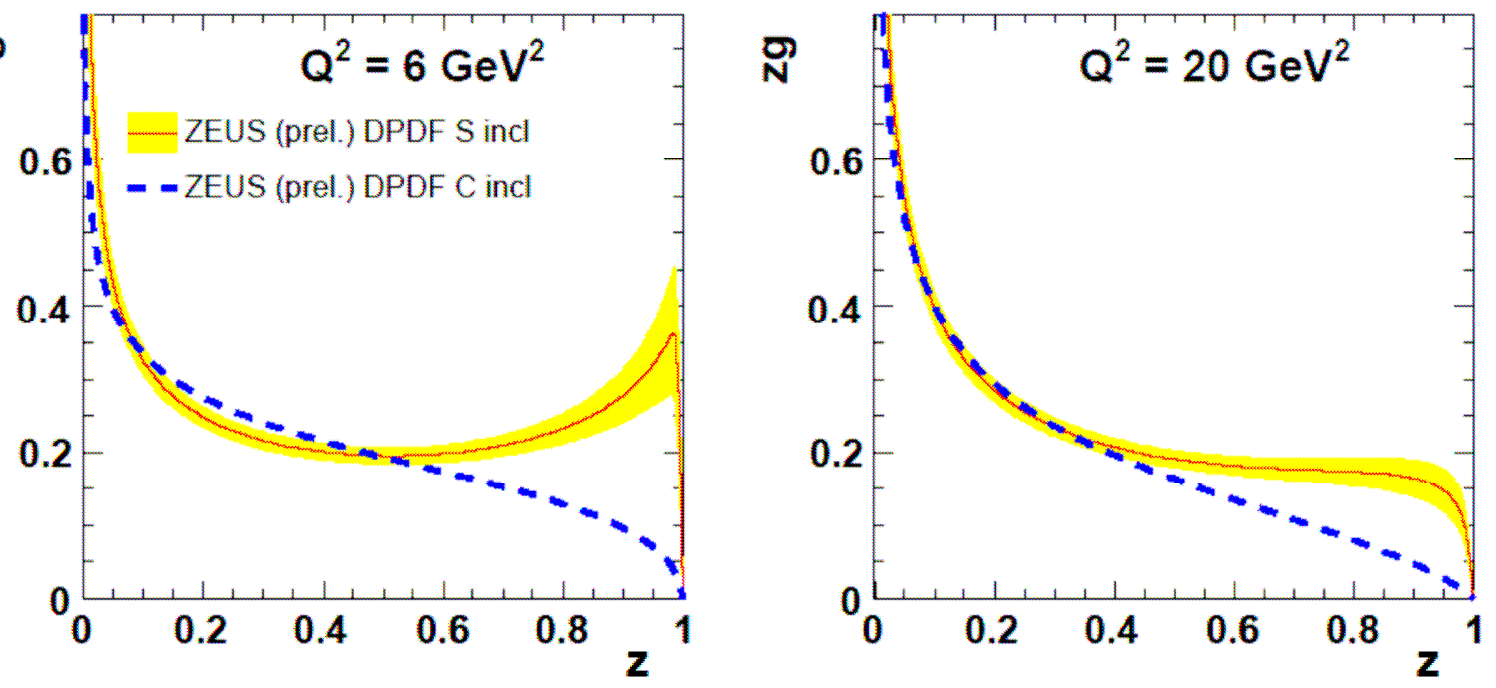

__ flexible gluons

-..- stiff gluons

Huge gluons uncertainty 


\section{Dijets in diffractive DIS \& PHP}

- Dijet production is directly sensitive to gluons

- photon-gluon fusion at LO

- DiJets in DIS (large Q2) (J.C. Collins 1998) factorisation holds in PQCD

- compare to predictions based on inclusive DIS fits

- use in incl+dijets fit

- DiJets in PHP $\left(Q^{2} \rightarrow 0\right)$ factorisation assumed for the resolved photon contribution

$$
\sigma\left(E_{\perp}^{2}, \ldots\right)=\sum_{j, k} f_{j}^{I P}\left(E_{\perp}^{2}\right) \otimes \sigma_{j k}(\ldots) \otimes f_{k}^{\gamma}\left(E_{\perp}^{2}\right)
$$

- strong suppression observed in pp collisions (CDF/Tevatron)

- compare to predictions based on incl+dijets fit 


\section{Dijets in DIS sensitive to gluons}

\section{ZEUS}
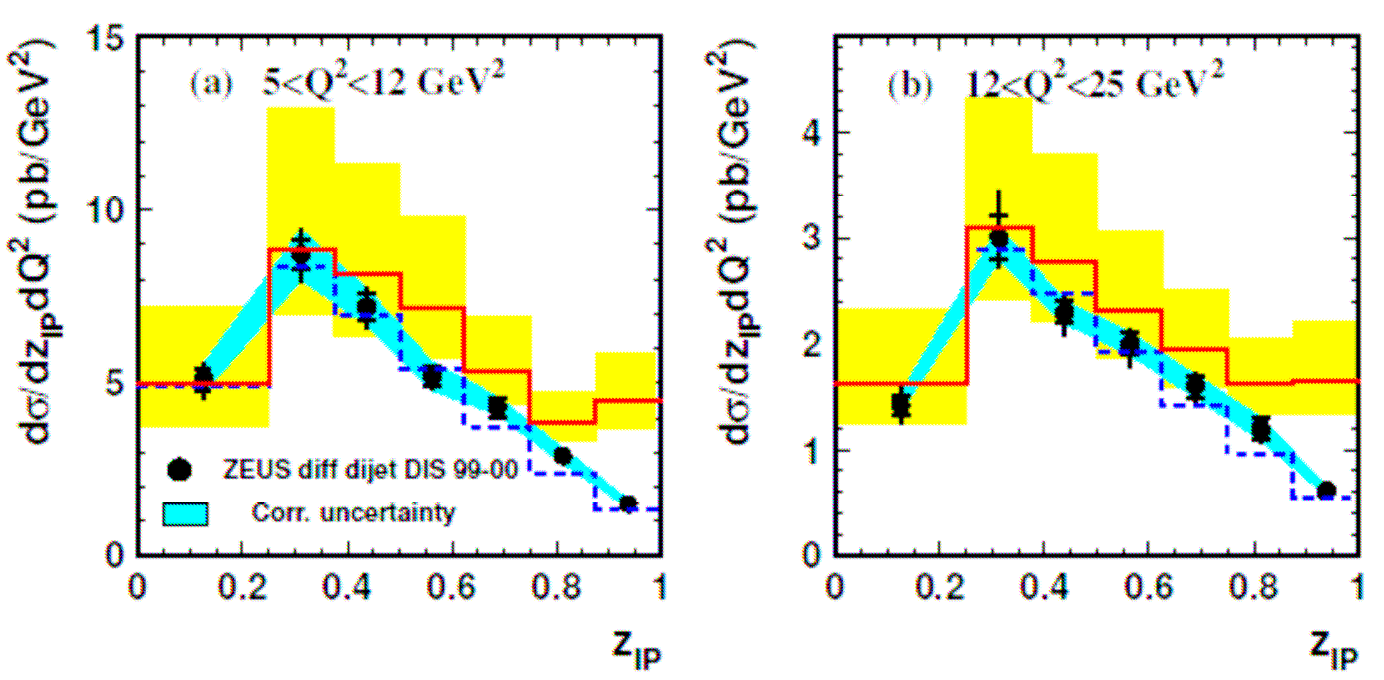

Fit $S$ fails at $z>0.4$

Fit C works

surprisingly well
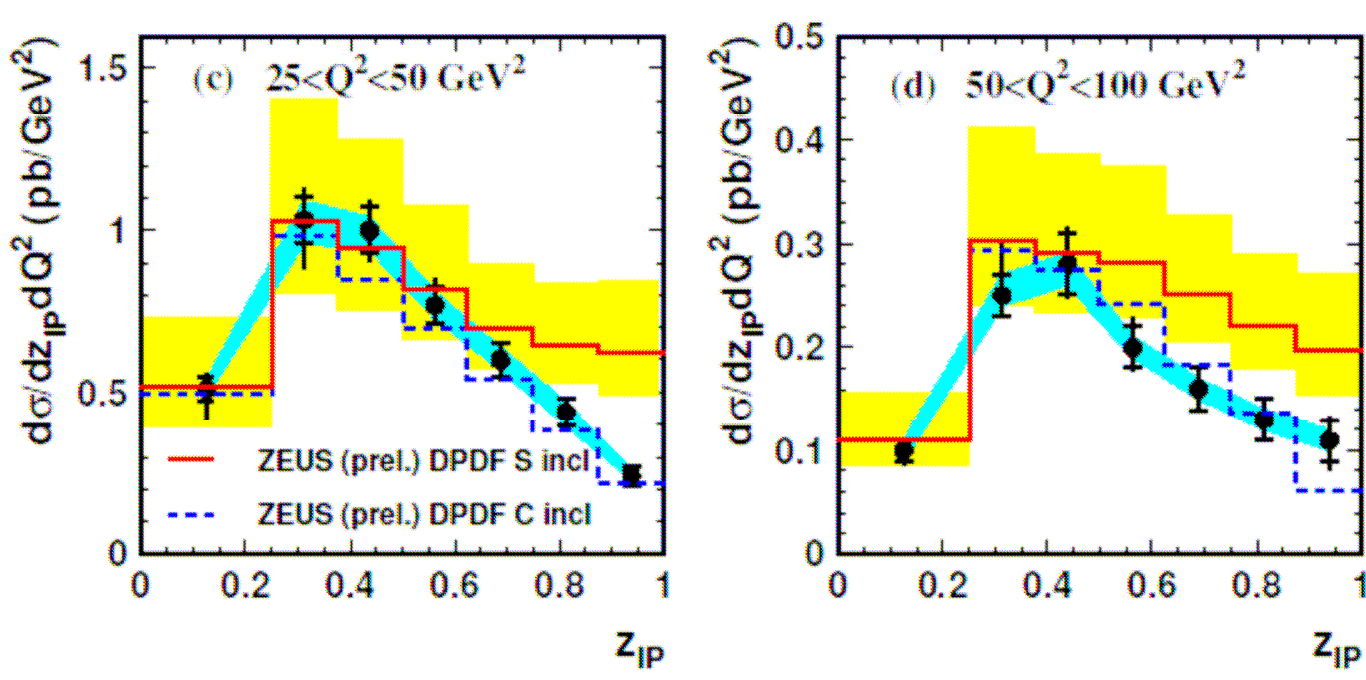

NLO QCD predictions from DISENT (Catani, Seymour) vs. ZEUS data

EPJ C52 (2007) 813

NLOJET++ (Nagy) results agree within 5\% 


\title{
Inclusive + DIS dijets fit
}

\section{ZEUS}
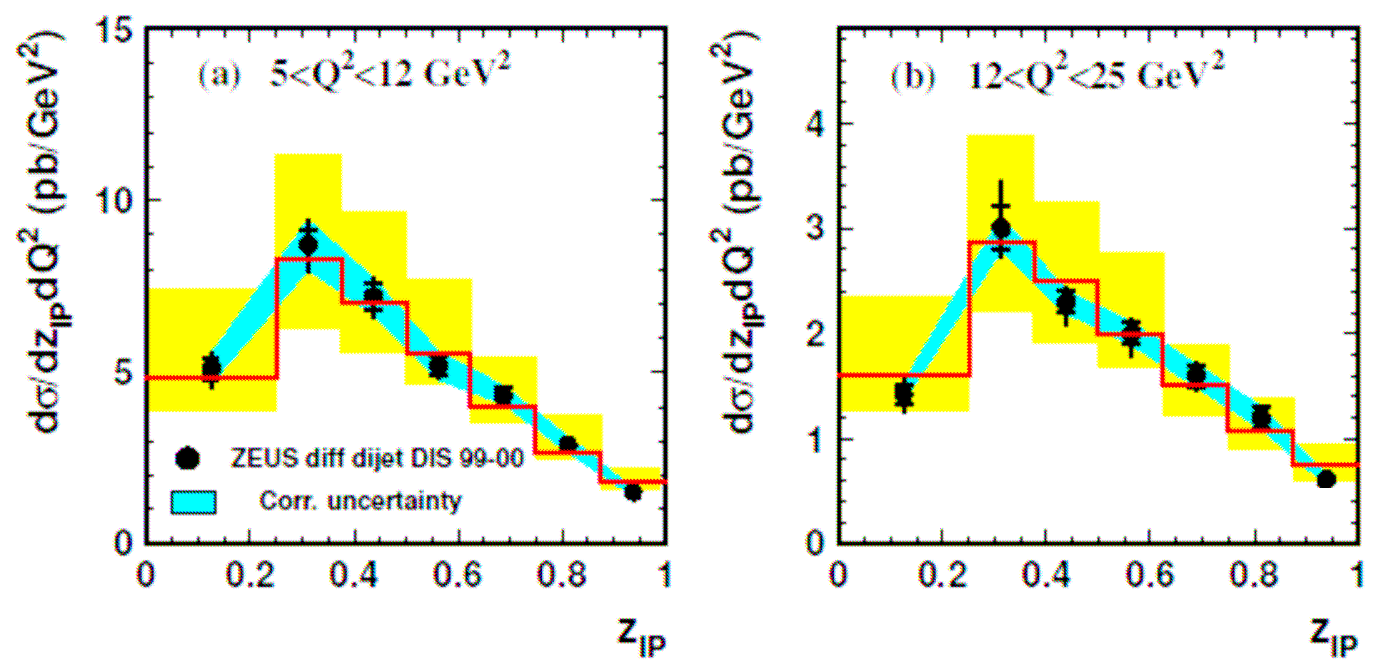

\author{
Fit S incl+dijets \\ good data description
}
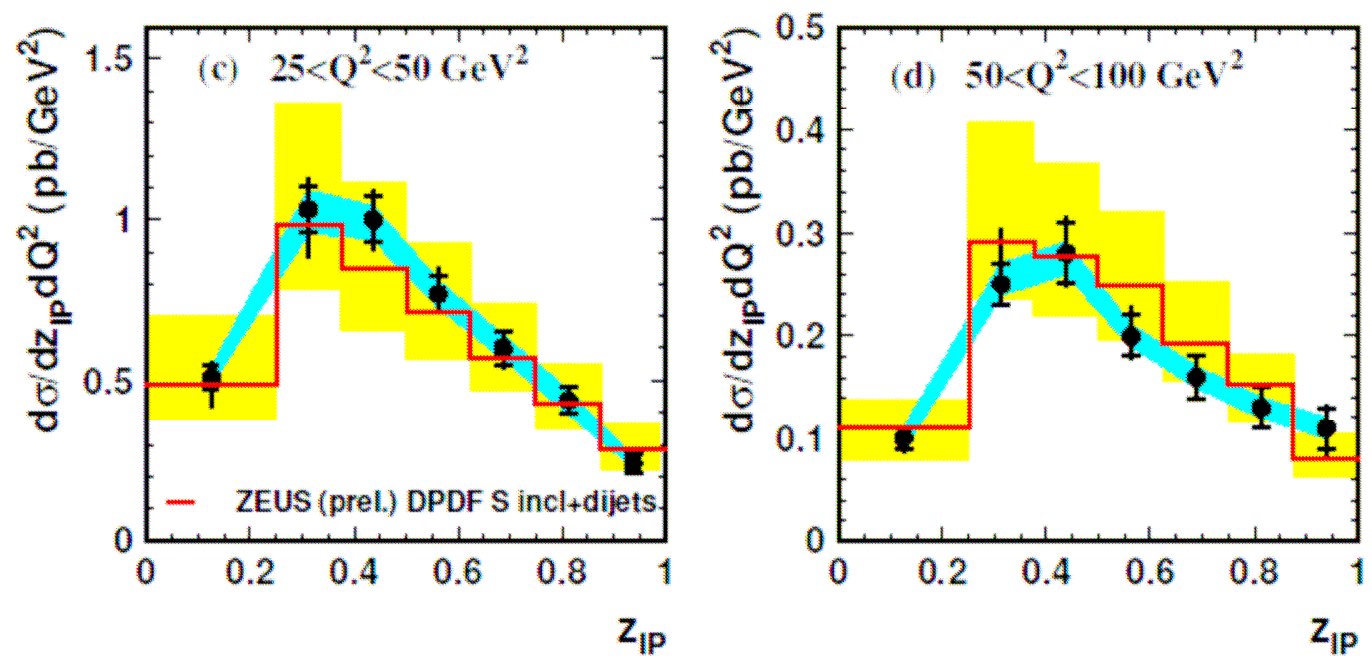


\section{DPDFs from the inclusive+dijets fit}

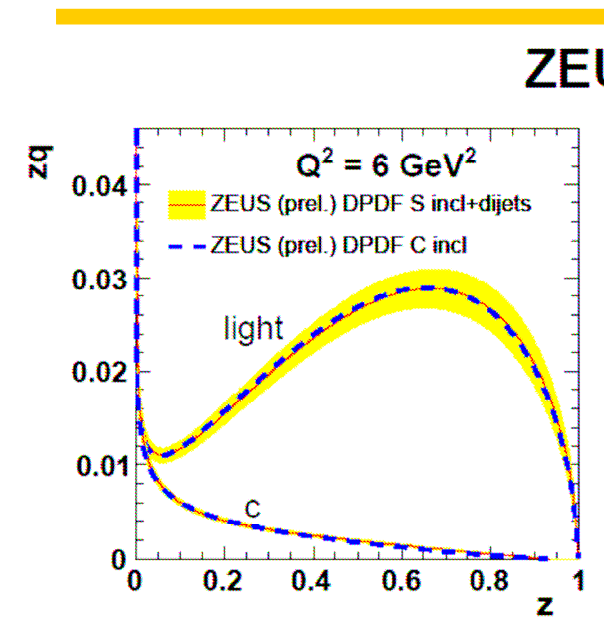

\section{ZEUS}
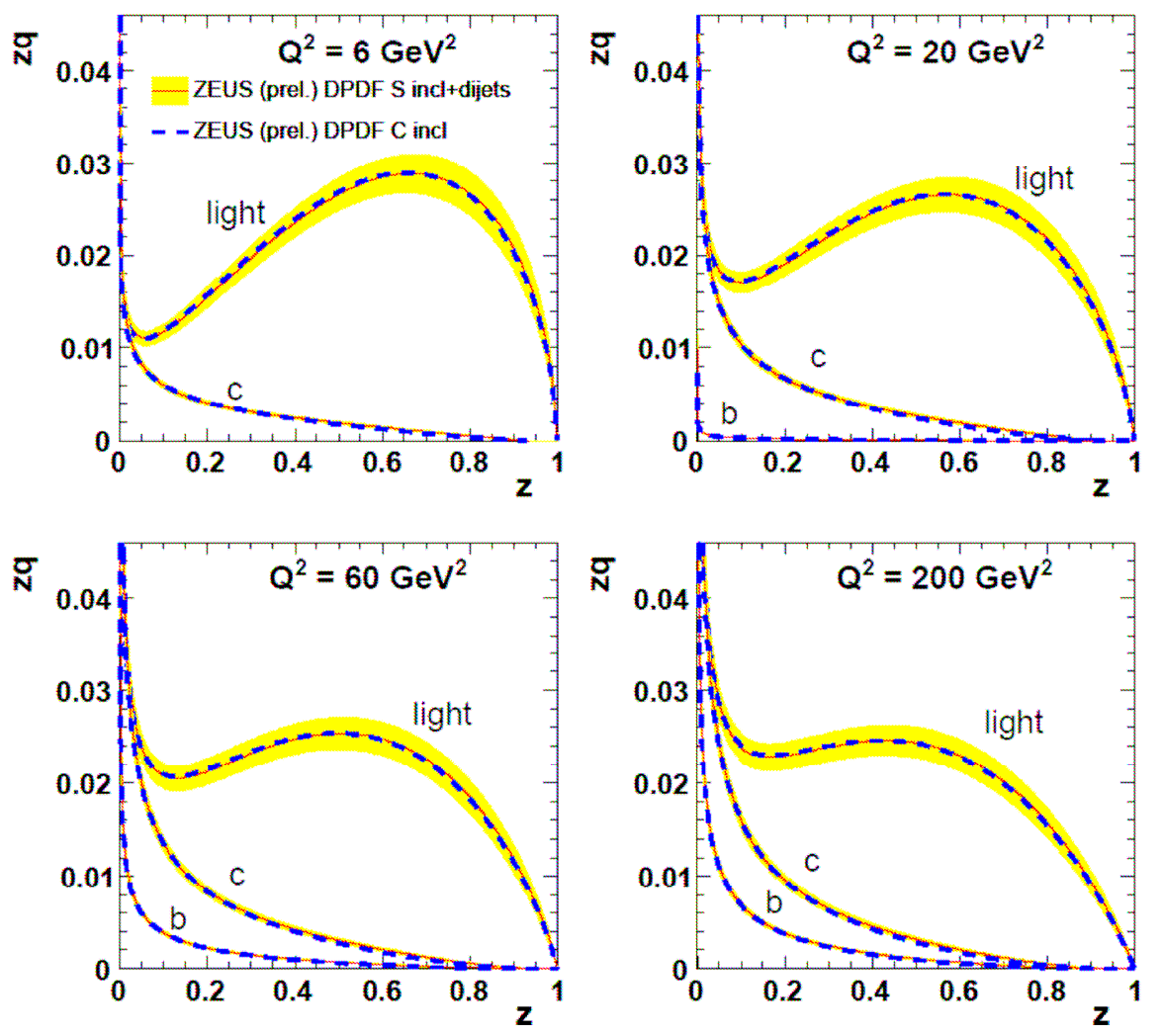

ZEUS
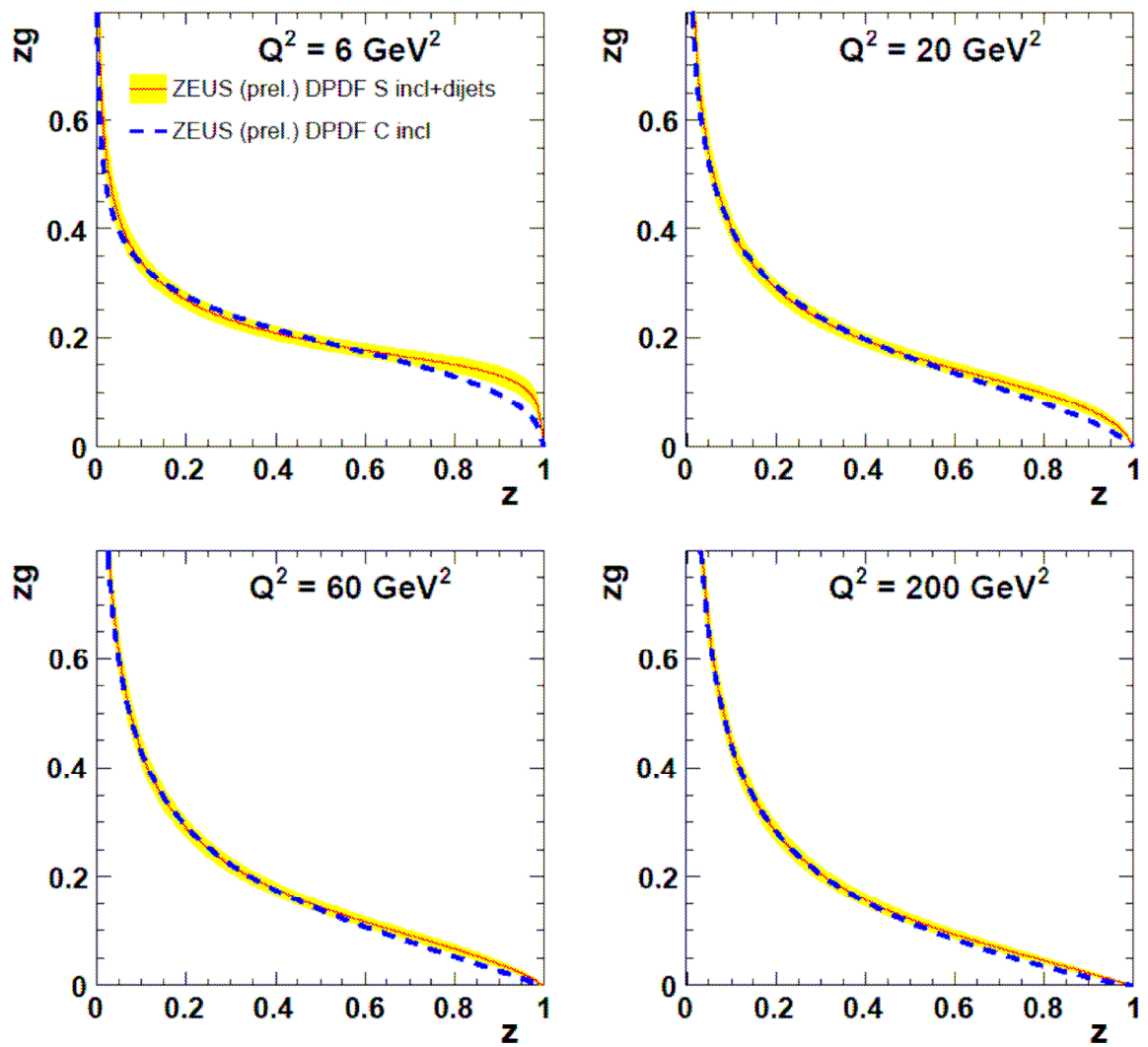

\section{— ZEUS (prel.) DPDF S incl+dijets \\ gluons fixed by dijet data}

- -ZEUS (prel.) DPDF C incl

\section{gluons guessed}




\section{Predictions for photoproduction of dijets vs. $x_{\gamma}$}

\section{ZEUS}

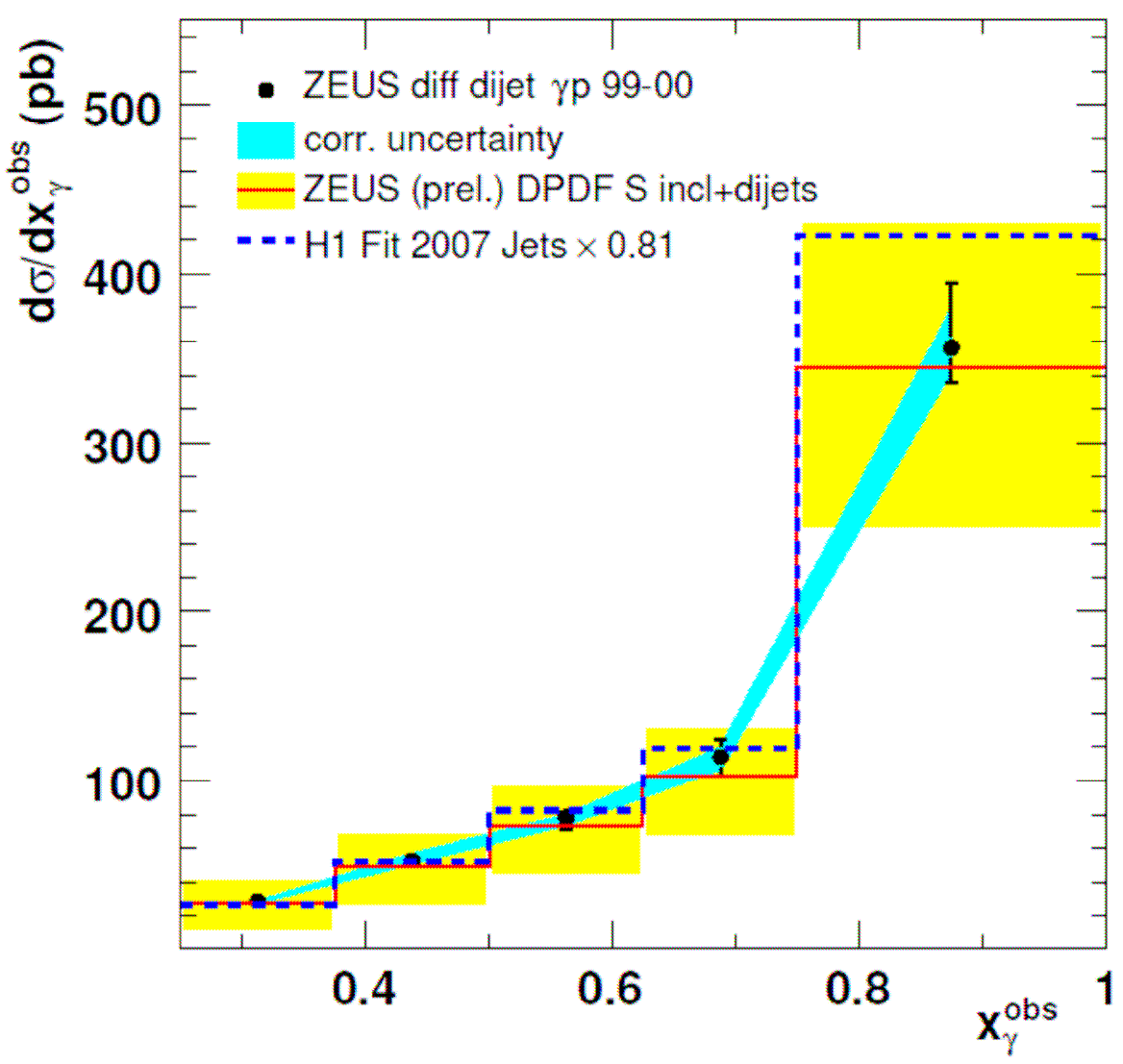

ZEUS data EPJ C55 (2008) 177

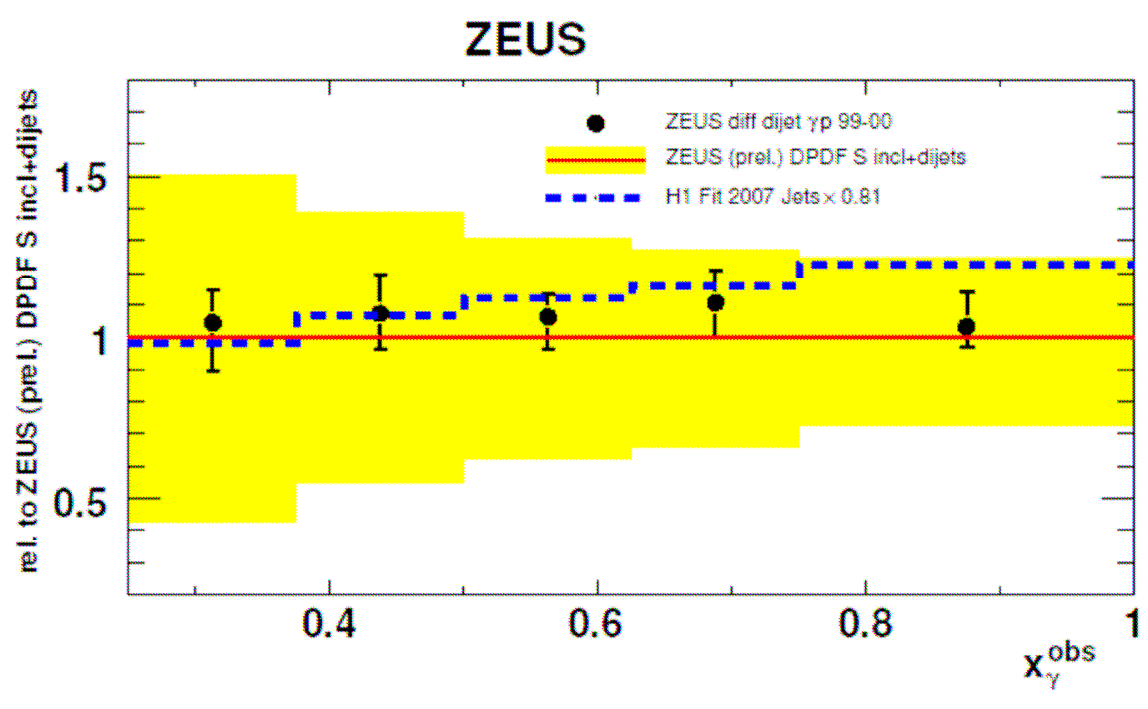

Very good data description No evidence for suppression

NLO QCD predictions obtained assuming factorisation Computer code by Frixione \& Ridolfi, $\gamma$ PDFs: GRV-HO 


\section{Predictions for photoproduction of dijets vs. $E_{T}$}
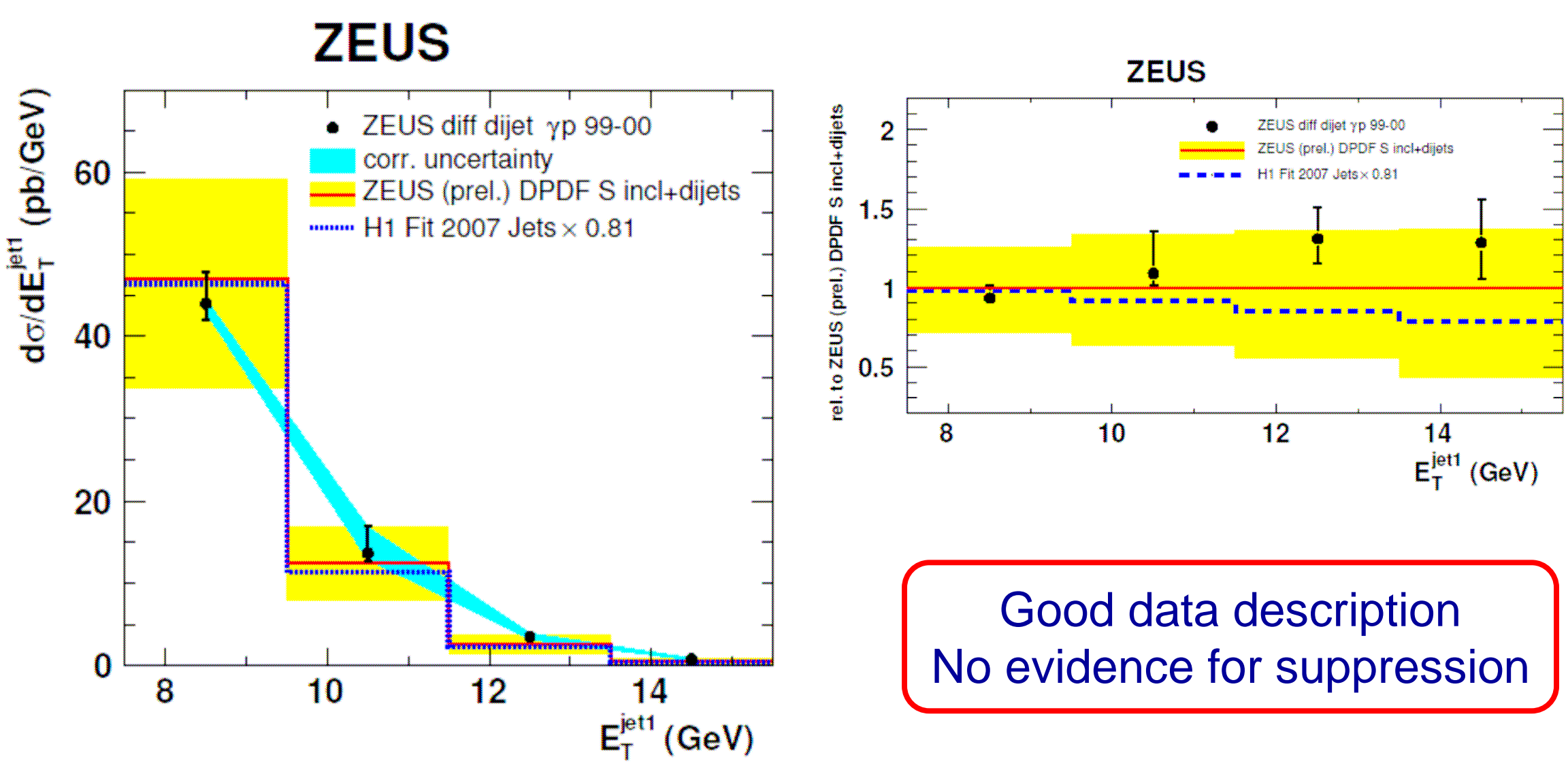

NLO QCD predictions obtained assuming factorisation Computer code by Frixione \& Ridolfi 


\section{Summary}

- A systematic NLO analysis of the ZEUS diffractive data performed

- Successful GM-VFNS (Thorne-Roberts) fits to

- inclusive DIS only

- inclusive DIS + DIS-dijets

- NLO predictions for dijet production, using new DPDFs agree very well with the data

- No evidence for suppression in photoproduction 


\section{EXTRAS}




\section{$Q_{0}^{2}, Q^{2}$ min and $\chi^{2}$ for inclusive fits}

Best $\chi^{2}$ at $Q_{0}^{2}=1.8 \mathrm{GeV}^{2}$

Data samples used in the fits:

$\mathrm{Q}_{\text {min }}^{2}>5 \mathrm{GeV}^{2}: 229(\mathrm{LRG})+36(\mathrm{LPS})=265$ points

$\mathrm{Q}^{2}{ }_{\text {min }}>10 \mathrm{GeV}^{2}: 179$ (LRG) +25 (LPS) $=204$ points

LPS: only $x_{I P}>0.02-$ to remove overlap with the LRG sample

Resulting $\chi^{2} / \mathrm{NDF}$

\begin{tabular}{|l|c|c|}
\hline & $\mathrm{Q}^{2}{ }_{\min }>5 \mathrm{GeV}^{2}$ & $\mathrm{Q}^{2}{ }_{\text {min }}>10 \mathrm{GeV}^{2}$ \\
\hline fit C & 1.18 & 1.03 \\
\hline fit S & 1.19 & 1.05 \\
\hline
\end{tabular}




\section{$Q_{\text {min }}^{2}-\chi^{2}$ dependence}

\section{ZEUS}

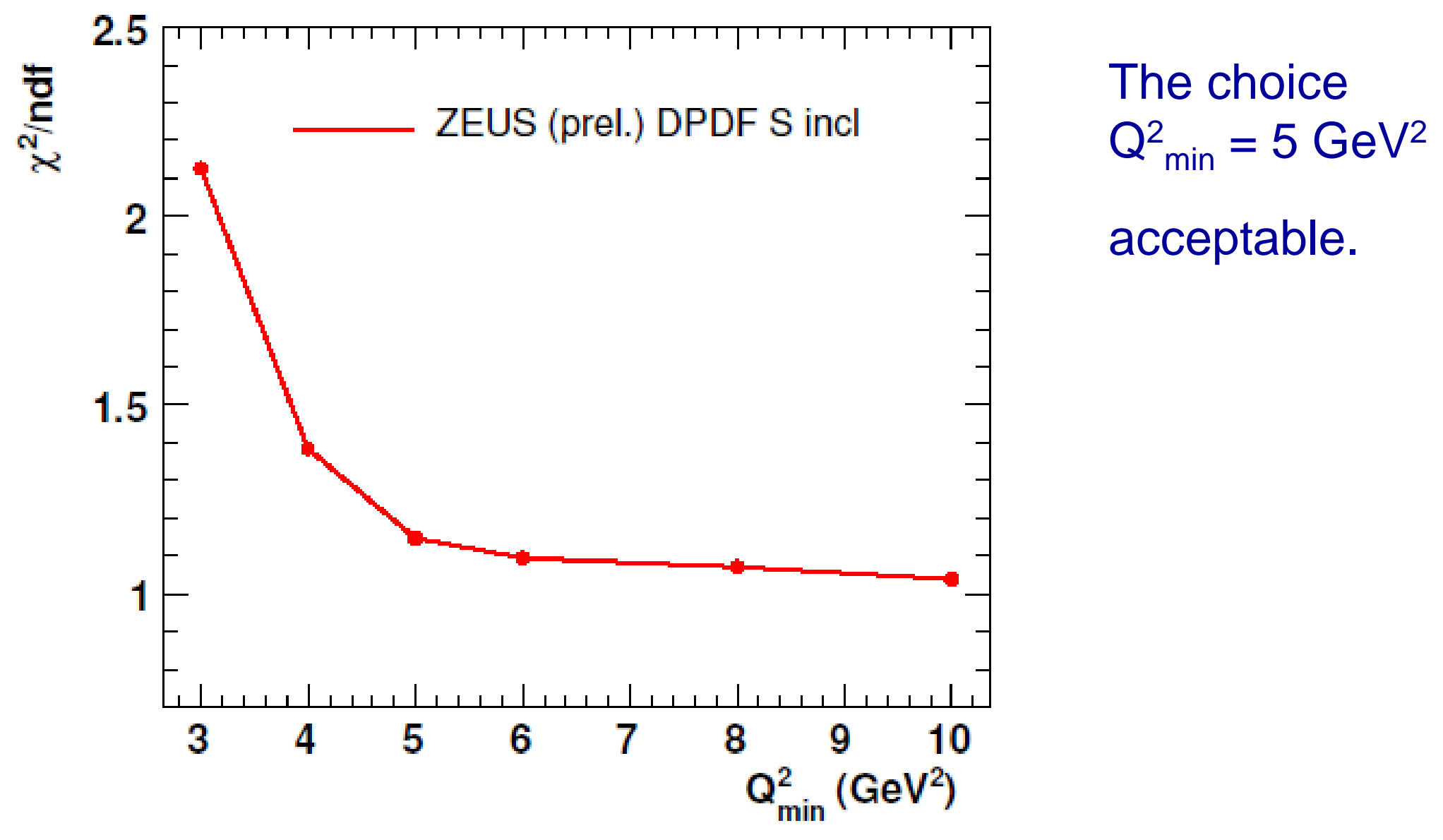




\section{$Q_{\text {min }}^{2}-D P D F s$ dependence}

\section{light quarks}

\section{ZEUS}
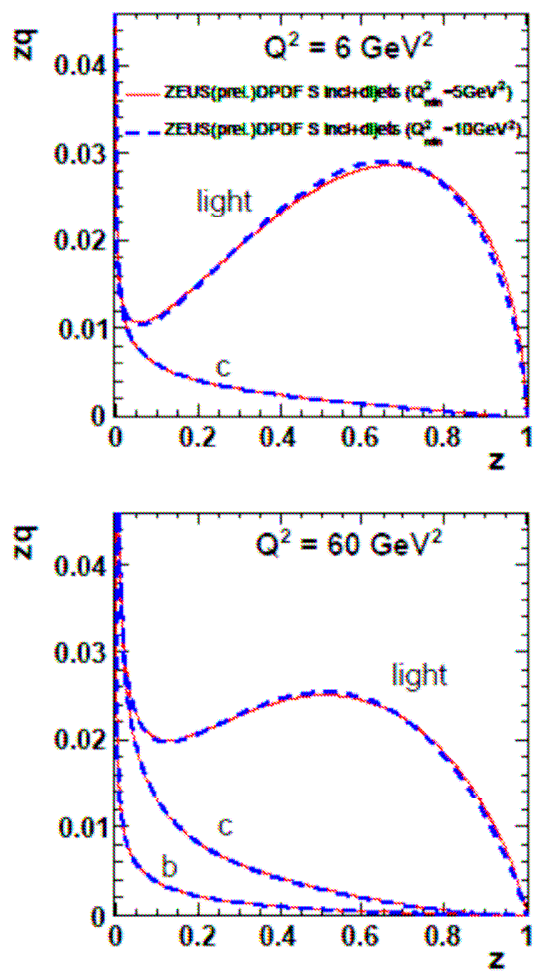
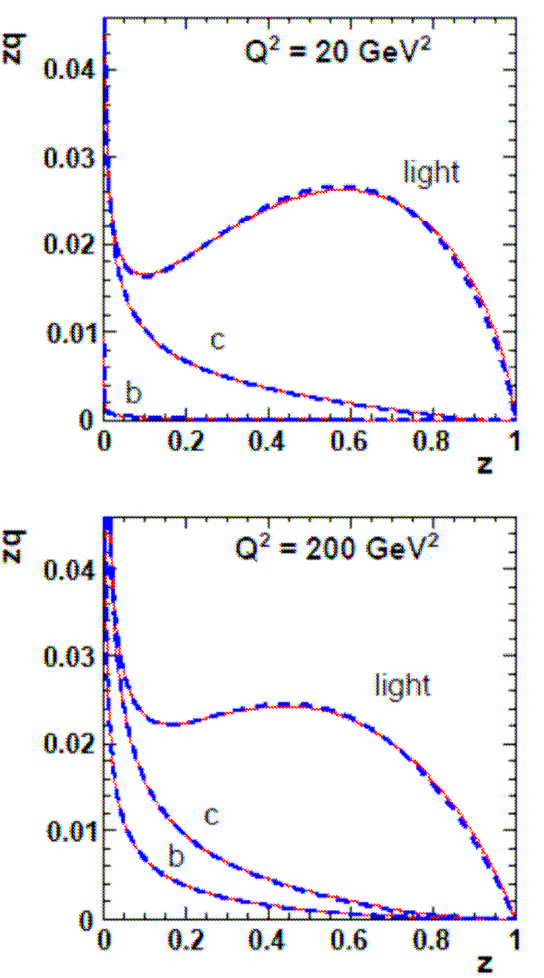

\section{gluons}
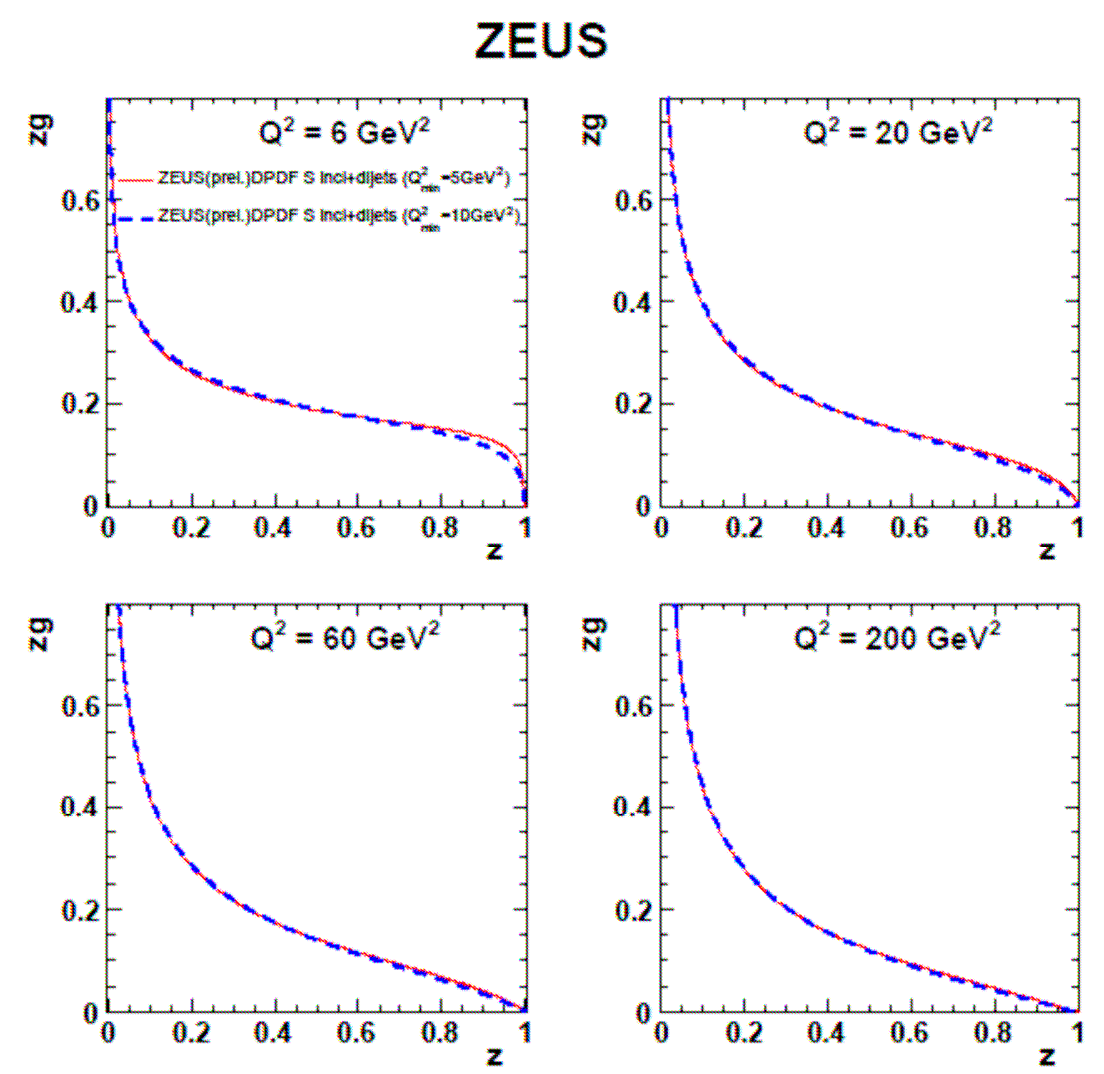

Very small difference between $\mathrm{Q}^{2}{ }_{\min }=5$ and $10 \mathrm{GeV}^{2}$ 